PROCEEDINGS OF THE

AMERICAN MATHEMATICAL SOCIETY

Volume 126, Number 3, March 1998, Pages 729-737

S $0002-9939(98) 04006-4$

\title{
SPECTRAL PROPERTIES OF CONTINUOUS REFINEMENT OPERATORS
}

\author{
R. Q. JIA, S. L. LEE, AND A. SHARMA
}

(Communicated by J. Marshall Ash)

\begin{abstract}
This paper studies the spectrum of continuous refinement operators and relates their spectral properties with the solutions of the corresponding
\end{abstract} continuous refinement equations.

\section{INTRODUCTION}

This paper studies the spectral properties of the operator $W_{c}: L^{p}(\mathbf{R}) \rightarrow L^{p}(\mathbf{R})$, $1 \leq p \leq \infty$, defined by

$$
\left(W_{c} f\right)(x):=\int_{\mathbf{R}} \alpha c(y) f(\alpha x-y) d y, \quad f \in L^{p}(\mathbf{R}),
$$

and its restriction to a certain invariant subspace. Here $\alpha>1$ is a dilation constant. An eigenfunction of $W_{c}$ with eigenvalue 1 is a solution in $L^{p}(\mathbf{R})$ of the functional equation

$$
\phi(x)=\int_{\mathbf{R}} \alpha c(y) \phi(\alpha x-y) d y,
$$

which is called a continuous refinement equation. The operator $W_{c}$ will be called a continuous refinement operator.

The simplest continuous refinement equation is one in which the kernel $c=$ $\frac{1}{2} \chi_{[-1,1]}$ and the dilation constant $\alpha=2$. Its solution with compact support is known as the up function, denoted by $u p(x)$, and is defined in terms of its Fourier transform

$$
\widehat{u p}(u)=\prod_{j=1}^{\infty} \frac{\sin \left(u 2^{-j}\right)}{u 2^{-j}} .
$$

The up function has interesting properties and important applications, and has been extensively studied by Russian mathematicians (see the survey articles [9], [10]). The up function corresponding to a dilation constant $\alpha>1$ and the kernel

$$
c=\frac{1}{2(\alpha-1)} \chi_{[-\alpha+1, \alpha-1]}
$$

Received by the editors October 25, 1995 and, in revised form, July 23, 1996.

1991 Mathematics Subject Classification. Primary 34K99, 41A15, 41A25, 41A30, 42C05, $42 \mathrm{C} 15$.

Key words and phrases. Continuous refinement equations, up function, continuous refinement operators, compact operators, spectrum, spectral radius, eigenvalues, dilation constant, power iteration. 
has also been studied independently by Kabaya and Iri [7], [8]. In [8] they gave a complete description of the spectrum of the corresponding refinement operator $W_{c}$ restricted to the space $L^{1}[-1,1]$. Recently, more general continuous refinement equations have been studied in conjunction with nonstationary subdivision processes and nonstationary multiresolution analyses (see [3], [2], [4], [5]). In this connection, one is interested in the existence and properties of the solution of (1.2) for a compactly supported kernel $c \in L^{p}(\mathbf{R})$, and also in the convergence of the power iteration $W_{c}^{n} \phi_{0}$. The operator $W_{c}$ is related to the adjoint of the continuous subdivision operator.

The convergence of the power iteration depends on the spectral radius of the refinement operator and is related to the existence and uniqueness of the solution of the continuous refinement equation. The object of this paper is to study the relationship between the spectrum of $W_{c}$ on one hand and the existence and uniqueness of the solution of the continuous refinement equation (1.2) and its properties on the other. We shall assume throughout this paper that $c$ is compactly supported with $\operatorname{supp}(c) \subset[-a, a]$, and, unless otherwise stated, $c \in L^{1}(\mathbf{R})$. In general $a$ depends on the dilation constant $\alpha$. We are interested in the compactly supported solution of the equation (1.2) and since it is an eigenfunction of $W_{c}$, we are led to consider the restriction of the operator to a subspace $L_{K}^{p}$ of $L^{p}(\mathbf{R})$ consisting of functions with supports in an interval $[-K, K]$. Then $L_{K}^{p}$ is an invariant subspace of $W_{c}$ if and only if

$$
K \geq \frac{a}{\alpha-1},
$$

a condition which we shall assume throughout the paper. Note that, in general, $a$ depends on $\alpha$. The restriction of $W_{c}$ to $L_{K}^{p}$ will be denoted by $W_{c, K}$. Thus

$$
\left(W_{c} f\right)(x):=\int_{-a}^{a} \alpha c(y) f(\alpha x-y) d y, \quad f \in L^{p}(\mathbf{R}),
$$

and

$$
\left(W_{c, K} f\right)(x):=\int_{-K}^{K} \alpha c(\alpha x-y) f(y) d y, \quad f \in L_{K}^{p} .
$$

In $\S 2$ we show that these operators are bounded and that $W_{c, K}$ is compact. The compactness of the operator $W_{c, K}$ is exploited to obtain its spectral properties. The main results are contained in $\S 3$. It is shown that if the kernel $c \in L^{1}(\mathbf{R})$ is compactly supported and normalized so that $\hat{c}(0)=1$, then the spectrum of $W_{c, K}$ is precisely the set

$$
\left\{1 / \alpha^{k}: k=0,1, \ldots\right\} \cup\{0\}
$$

and all the nonzero elements in the spectrum are simple eigenvalues of $W_{c, K}$. These results extend those in [8] to a general kernel $c$ and to continuous refinement operators on $L_{K}^{p}$ for $1 \leq p \leq \infty$. Further, the method here is simpler and more general, and using the same method the results can be further extended to the multivariate setting.

It follows from the spectral properties of $W_{c, K}$ that the continuous refinement equation (1.2) has a unique solution if and only if $\hat{c}(0)=1$, and the power iteration $W_{c, K}^{n} \phi_{0}$ converges to the solution for any normalized initial function $\phi_{0}$. Further, the solution is infinitely differentiable. The adjoint operator $W_{c}^{*}$ is related to the continuous subdivision operator (see [4]). It is studied in $\S 4$. 
We remark that our results on the solution of the continuous refinement equation and the convergence of the power iteration of the continuous refinement operator and the continuous subdivision operator are obtained via the spectral properties of $W_{c, K}$ without recourse to the Paley-Wiener theorem. In one-dimension they are more general than most of the results in [4] and [3]. We also remark that our approach depends on the fact that $W_{c}$ has an invariant subspace consisting of functions with supports in a compact set. This property is not available for the continuous subdivision operator.

\section{The OPERATORS $W_{c}$ AND $W_{c, K}$}

The operator $W_{c}$, and hence $W_{c, K}$, can be expressed as a convolution-dilation operator,

$$
\left(W_{c} f\right)(x):=\alpha(c * f)(\alpha x), \quad f \in L^{p}(\mathbf{R}) .
$$

Taking the Fourier transforms of the functions in (2.1), it is equivalent to

$$
\left(W_{c} f\right)^{\wedge}(u):=\hat{c}(u / \alpha) \hat{f}(u / \alpha), \quad f \in L^{p}(\mathbf{R}) .
$$

Proposition 2.1. Let $1 \leq p \leq \infty$ and $1 / p+1 / q=1$. If $c \in L^{1}(\mathbf{R})$, the operator $W_{c}$ is a bounded linear operator on $L^{p}(\mathbf{R})$, and

$$
\left\|W_{c}\right\| \leq \alpha^{\frac{1}{q}}\|c\|_{1} .
$$

Proof. For any $f \in L^{p}(\mathbf{R})$,

$$
W_{c} f(x)=\alpha c * f(\alpha x), \quad x \in \mathbf{R},
$$

and

$$
\begin{aligned}
\left\|W_{c} f\right\|_{p} & =\left\{\int_{\mathbf{R}}|\alpha(c * f)(\alpha x)|^{p} d x\right\}^{1 / p} \\
& =\left\{\alpha^{p-1} \int_{\mathbf{R}}|(c * f)(x)|^{p} d x\right\}^{1 / p} \\
& =\alpha^{\frac{1}{q}}\|c * f\|_{p} .
\end{aligned}
$$

By Young's inequality,

$$
\left\|W_{c} f\right\|_{p} \leq \alpha^{\frac{1}{q}}\|c\|_{1}\|f\|_{p}, \quad \text { for all } f \in L^{p}(\mathbf{R}) .
$$

Hence, $W_{c}$ is bounded and (2.3) holds.

It follows from Proposition 2.1 that $W_{c, K}$ is also a bounded linear operator on $L_{K}^{p}$. We shall show that $W_{c, K}$ is a compact operator.

Lemma 2.1. For $f \in L_{K}^{p}, 1 \leq p<\infty$, and $h>0$, define

$$
f^{h}(x):=\frac{1}{2 h} \int_{x-h}^{x+h} f(t) d t, \quad x \in[-K, K],
$$

and $f^{h}(x)=0$ otherwise. Then

$$
\begin{gathered}
f^{h} \in L_{K}^{p} \quad \text { and } \quad\left\|f^{h}\right\|_{p} \leq\|f\|_{p}, \\
\left\|f^{h}-f\right\|_{p} \rightarrow 0 \quad \text { as } h \rightarrow 0,
\end{gathered}
$$

and

$$
\left(W_{c, K} f\right)^{h}=W_{c^{\alpha h}, K} f .
$$


Proof. The relations (2.6) and (2.7) are well-known (see [1]). To prove (2.8), note that

$$
\begin{aligned}
\left(W_{c, K} f\right)^{h}(x) & =\frac{1}{2 h} \int_{x-h}^{x+h} W_{c, K} f(t) d t \\
& =\frac{1}{2 h} \int_{x-h}^{x+h} \int_{-K}^{K} \alpha c(\alpha t-y) f(y) d y d t .
\end{aligned}
$$

Applying Fubini's Theorem a straightforward calculation gives (2.8).

Proposition 2.2. If $c \in L^{1}[-a, a]$, the operator $W_{c, K}$ is a compact operator on $L_{K}^{p}$, for $1 \leq p \leq \infty$.

Proof. Let $\left\{f_{n}\right\}$ be a bounded sequence in $L_{K}^{p}$, and suppose that $\left\|f_{n}\right\|_{p} \leq M$. By Young's inequality,

$$
\left\|W_{c, K} f_{n}\right\|_{p} \leq \alpha^{\frac{1}{q}}\|c\|_{1}\left\|f_{n}\right\|_{p}, \quad \text { for all } n .
$$

Therefore, $\left\{W_{c, K} f_{n}\right\}$ is a bounded sequence in $L_{K}^{p}$.

We now show that $\left\{W_{c, K} f_{n}\right\}$ is equicontinuous in $L_{K}^{p}$ for $1 \leq p<\infty$. By Young's inequality and (2.8),

$$
\left\|\left(W_{c, K} f_{n}\right)^{h}-W_{c, K} f_{n}\right\|_{p} \leq \alpha^{\frac{1}{q}}\left\|c^{\alpha h}-c\right\|_{1}\left\|f_{n}\right\|_{p} \leq \alpha^{\frac{1}{q}} M\left\|c^{\alpha h}-c\right\|_{1} \text {, for all } n .
$$

By (2.7), for any $\varepsilon>0$, there exists a $\delta>0$, such that

$$
\left\|c^{\alpha h}-c\right\|_{1}<\frac{\varepsilon}{\alpha^{\frac{1}{q}} M},
$$

for $0<h<\delta$. Hence,

$$
\left\|\left(W_{c, K} f_{n}\right)^{h}-W_{c, K} f_{n}\right\|_{p}<\varepsilon,
$$

for $0<h<\delta$. By the Kolmogorov-Tulajkov Theorem ([1], p. 216), $\left\{W_{c, K} f_{n}\right\}$ has a subsequence which converges in $L_{K}^{p}$. Thus $W_{c, K}$ is a compact operator on $L_{K}^{p}$.

If $p=\infty$, then $W_{c, K} f_{n}$ is continuous for each $n$. Therefore $\left\{W_{c, K} f_{n}\right\}$ is a bounded sequence in $C([-K, K])$. A similar argument as above shows that $\left\{W_{c, K} f_{n}\right\}$ is equicontinuous. By Ascoli's Theorem, $\left\{W_{c, K} f_{n}\right\}$ is a relatively compact set in $C([-K, K])$. Therefore, $W_{c, K}$ is a compact operator on $L_{K}^{\infty}$.

\section{Spectrum of $W_{c, K}$}

In this section we give a complete description of the spectrum of the compact operator $W_{c, K}$, and deduce properties of the solutions of the continuous refinement equation from the spectral properties of $W_{c, K}$. Let $\sigma_{p}\left(W_{c, K}\right)$ denote the spectrum of $W_{c, K}: L_{K}^{p} \rightarrow L_{K}^{p}$ and let $r_{p}\left(W_{c, K}\right)$ denote its spectral radius.

Lemma 3.1. Suppose $\hat{c}(0)=1$. If $\lambda \neq 0$ is an eigenvalue of $W_{c, K}$, then $\lambda=1 / \alpha^{k}$ for some nonnegative integer $k$.

Proof. Let $f$ be an eigenfunction of $W_{c, K}$ corresponding to the eigenvalue $\lambda$. Taking the Fourier transforms of the functions on both sides of the equation $W_{c, K} f=\lambda f$, we have

$$
\hat{c}(u / \alpha) \hat{f}(u / \alpha)=\lambda \hat{f}(u), \quad u \in \mathbf{R} .
$$

Suppose $\hat{f}(0)=\cdots=\hat{f}^{(k-1)}(0)=0$ but $\hat{f}^{(k)}(0) \neq 0$. Then $\hat{f}(u)=u^{k} g(u), u \in \mathbf{R}$, for some analytic function $g$ with $g(0) \neq 0$. Equation (3.1) becomes

$$
\hat{c}(u / \alpha) u^{k} g(u / \alpha) / \alpha^{k}=\lambda u^{k} g(u) .
$$


It follows that

$$
\hat{c}(u / \alpha) g(u / \alpha)=\alpha^{k} \lambda g(u) .
$$

Taking the limit as $u \rightarrow 0$ in the above equation, we obtain $g(0)=\alpha^{k} \lambda g(0)$. Therefore $\alpha^{k} \lambda=1$, and hence $\lambda=1 / \alpha^{k}$, as desired.

Proposition 3.1. Suppose that $\hat{c}(0)=1$, and let $k$ be a nonnegative integer. If $\phi$ is a nontrivial function in $L_{K}^{p}$ such that $\left(W_{c, K}-1 / \alpha^{k}\right)^{m} \phi=0$ for some positive integer $m$, then

$$
\hat{\phi}(0)=\cdots=\hat{\phi}^{(k-1)}(0)=0 \quad \text { and } \quad \hat{\phi}^{(k)}(0) \neq 0 .
$$

Consequently, any nonzero eigenvalue of $W_{c, K}$ is simple.

Proof. Let $j$ be the smallest nonnegative integer such that $\hat{\phi}^{(j)}(0) \neq 0$. Then $\hat{\phi}$ can be written as $\hat{\phi}(u)=u^{j} g(u), u \in \mathbf{R}$, where $g$ is an analytic function with $g(0) \neq 0$. For $n=1,2, \ldots, m$, let $\phi_{n}:=\left(W_{c, K}-1 / \alpha^{k}\right)^{n} \phi$. Taking the Fourier transforms of the functions on both sides of $\phi_{1}=\left(W_{c, K}-1 / \alpha^{k}\right) \phi$ leads to

$$
\begin{aligned}
\hat{\phi}_{1}(u) & =\hat{c}(u / \alpha) \hat{\phi}(u / \alpha)-\hat{\phi}(u) / \alpha^{k} \\
& =u^{j}\left(\hat{c}(u / \alpha) g(u / \alpha) / \alpha^{j}-g(u) / \alpha^{k}\right), \quad u \in \mathbf{R} .
\end{aligned}
$$

It follows that if $j \neq k$, then $\hat{\phi}_{1}^{(j)}(0) \neq 0$. Since $\phi_{n}=\left(W_{c, K}-1 / \alpha^{k}\right) \phi_{n-1}$, an inductive argument shows that if $j \neq k$, then $\hat{\phi}_{n}^{(j)}(0) \neq 0$ for all $n=1,2, \ldots, m$. Therefore, $\left(W_{c, K}-1 / \alpha^{k}\right)^{m} \phi=0$ implies that $j=k$, i.e., (3.2) holds.

Now suppose that $\lambda \neq 0$ is an eigenvalue of $W_{c, K}$. Then by Lemma $3.1, \lambda=1 / \alpha^{k}$ for some nonnegative integer $k$. Let $\phi \in L_{K}^{p}$ be the corresponding eigenfunction, and let $\varphi \in L_{K}^{p}$ be a nontrivial function such that $\left(W_{c, K}-1 / \alpha^{k}\right)^{m} \varphi=0$ for some positive integer $m$. Then (3.2) also holds for $\varphi$. Let $\psi:=\varphi-t \phi$, where $t:=\hat{\varphi}^{(k)}(0) / \hat{\phi}^{(k)}(0)$. Then $\left(W_{c, K}-1 / \alpha^{k}\right)^{m} \psi=0$. But $\hat{\psi}(0)=\cdots=\hat{\psi}^{(k-1)}(0)=\hat{\psi}^{(k)}(0)=0$, and by what has been just proved, we must have $\psi=0$. It follows that $\varphi=t \phi$. Therefore, the eigenvalue $1 / \alpha^{k}$ is simple.

Theorem 3.1. Suppose $\phi_{0}$ is a function in $L_{K}^{p}$ such that

$$
\hat{\phi}_{0}(0)=\cdots=\hat{\phi}_{0}^{(k-1)}(0)=0 \quad \text { and } \quad \hat{\phi}_{0}^{(k)}(0) \neq 0 .
$$

Then $\left(\alpha^{k} W_{c, K}\right)^{n} \phi_{0}$ converges in the $L_{p}$-norm to an eigenfunction of $W_{c, K}$ corresponding to the eigenvalue $1 / \alpha^{k}$.

Proof. For $k=0,1,2, \ldots$, let

$$
N_{k}:=\left\{f \in L_{K}^{p}: \hat{f}(0)=\cdots=\hat{f}^{(k-1)}(0)=0\right\} .
$$

Then $N_{k}$ is an invariant subspace of $W_{c, K}$. By Proposition 3.1, $\sigma_{p}\left(\left.W_{c, K}\right|_{N_{k+1}}\right)$ does not contain $1 / \alpha^{j}$ for $j=0, \ldots, k$. Hence

$$
r_{p}\left(\left.W_{c, K}\right|_{N_{k+1}}\right) \leq 1 / \alpha^{k+1} .
$$

Let $\psi:=\alpha^{k} W_{c, K} \phi_{0}-\phi_{0}$. By the assumption on $\phi_{0}$, we have $\hat{\phi}_{0}(u)=u^{k} g(u)$, $u \in \mathbf{R}$, for some analytic function $g$ with $g(0) \neq 0$. Hence

$$
\hat{\psi}(u)=\alpha^{k} \hat{\phi}_{0}(u / \alpha) \hat{c}(u / \alpha)-\hat{\phi}_{0}(u)=u^{k}(g(u / \alpha) \hat{c}(u / \alpha)-g(u)) .
$$


It follows that $\hat{\psi}(0)=\cdots=\hat{\psi}^{(k)}(0)=0$. In other words, $\psi \in N_{k+1}$. From (3.4) we see that there exist constants $C>0$ and $r, 0<r<1$, such that

$$
\left\|\left(W_{c, K}\right)^{n} \psi\right\|_{p} \leq C\left(r / \alpha^{k}\right)^{n}, \quad n=1,2, \ldots .
$$

Therefore we have

$$
\left\|\left(\alpha^{k} W_{c, K}\right)^{n+1} f-\left(\alpha^{k} W_{c, K}\right)^{n} f\right\|_{p}=\left\|\left(\alpha^{k} W_{c, K}\right)^{n} \psi\right\|_{p} \leq C r^{n} .
$$

This shows that $\left(\alpha^{k} W_{c, K}\right)^{n} f$ converges in the $L_{p}$-norm to some function $\phi \in L_{K}^{p}$. Clearly, $\phi$ is an eigenfunction of $W_{c, K}$ corresponding to the eigenvalue $1 / \alpha^{k}$.

Corollary 3.1. Suppose $c \in L^{1}(\mathbf{R})$ is compactly supported and $\hat{c}(0)=1$. Then

$$
\sigma_{p}\left(W_{c, K}\right)=\left\{1 / \alpha^{k}: k=0,1, \ldots\right\} \cup\{0\} .
$$

The nonzero elements of $\sigma_{p}\left(W_{c, K}\right)$ are simple eigenvalues of $W_{c, K}$. Further, 0 is not an eigenvalue of $W_{c, K}$.

Proof. The result (3.5) and the simplicity of the eigenvalues follow from Lemma 3.1, Proposition 3.1 and Theorem 3.1.

To show that 0 is not an eigenvalue of $W_{c, K}$, suppose that $f \in L_{K}^{p}$ such that $W_{c, K} f=0$. Then

$$
\hat{c}(u) \hat{f}(u)=0, \quad \text { for all } u \in \mathbf{R} .
$$

Since $\hat{c}$ is entire and $\hat{c}(0)=1$, there is a positive number $\delta$ such that $\hat{c}(z) \neq 0$ for all $|z|<\delta$. Hence, $\hat{f}(z)=0$ for all $|z|<\delta$. It follows that $\hat{f}=0$, and hence $f=0$. Therefore, 0 is not an eigenvalue of $W_{c, K}$.

Corollary 3.2. Suppose that $c \in L^{1}(\mathbf{R})$ is compactly supported. The continuous refinement equation (1.2) has a unique nontrivial solution in $L_{K}^{p}$ if and only if $\hat{c}(0)=1$.

Theorem 3.2. Suppose that $c \in L^{1}(\mathbf{R})$ with $\operatorname{supp}(c) \subset[-a, a]$ and $\hat{c}(0)=1$. Let $\phi \in L_{K}^{p}(\mathbf{R})$ be the solution of the continuous refinement equation $(1.2)$ with $\hat{\phi}(0)=$ 1. Then $\phi \in C^{\infty}(\mathbf{R})$ and, for each positive integer $k, \phi^{(k)}$ is an eigenfunction of $W_{c, K}$ corresponding to the eigenvalue $1 / \alpha^{k}$.

Further $\operatorname{supp}(\phi) \subset[-a /(\alpha-1), a /(\alpha-1)]$.

Proof. Taking the Fourier transforms of the functions on both sides of the equation $W_{c, K} \phi=\phi$, we obtain $\hat{c}(u / \alpha)=\hat{\phi}(u) / \hat{\phi}(u / \alpha)$. It follows that

$$
\prod_{j=1}^{n} \hat{c}\left(u / \alpha^{j}\right)=\hat{\phi}(u) / \hat{\phi}\left(u / \alpha^{n}\right), \quad u \in \mathbf{R} .
$$

Since $\hat{\phi}(0)=1$, this shows that $\prod_{j=1}^{n} \hat{c}\left(u / \alpha^{j}\right)$ converges to $\hat{\phi}(u)$ for each $u \in \mathbf{R}$.

Let $\varphi$ be an eigenfunction of $W_{c, K}$ corresponding to the eigenvalue $1 / \alpha^{k}$. By Proposition 3.1, $\hat{\varphi}(u)=u^{k} g(u), u \in \mathbf{R}$, for some analytic function $g$ with $g(0) \neq 0$. Since $\varphi=\alpha^{k} W_{c, K} \varphi$, we have $\hat{\varphi}(u)=\alpha^{k} \hat{c}(u / \alpha) \hat{\varphi}(u / \alpha)$. Iterating $n$ times gives

$$
\hat{\varphi}(u)=\alpha^{n k} \hat{\varphi}\left(u / \alpha^{n}\right) \prod_{j=1}^{n} \hat{c}\left(u / \alpha^{j}\right)=u^{k} g\left(u / \alpha^{n}\right) \prod_{j=1}^{n} \hat{c}\left(u / \alpha^{j}\right) .
$$

Taking the limit as $n \rightarrow \infty$ gives

$$
\hat{\varphi}(u)=g(0) u^{k} \hat{\varphi}(u), \quad u \in \mathbf{R} .
$$


Hence the $k$-order derivative $\phi^{(k)}$ of $\phi$ exists and $\varphi=t \varphi^{(k)}$ for some $t \in \mathbf{C}$. Since this holds for all $k=0,1, \ldots$, it follows that $\phi \in C^{\infty}(\mathbf{R})$.

Note that if $\phi_{0}$ is continuous and $\operatorname{supp}\left(\phi_{0}\right) \subset[-K, K]$, then $\phi_{n}$ is continuous and $\operatorname{supp}\left(\phi_{n}\right) \subset\left[-l_{n}, l_{n}\right]$, for $n=1,2, \ldots$, where

$$
l_{n}:=\frac{a \alpha^{n-1}+a \alpha^{n-2}+\cdots+K}{\alpha^{n}}=\frac{a\left(\alpha^{n}-1\right)+K(\alpha-1)}{(\alpha-1) \alpha^{n}} .
$$

Since $\phi_{n}$ converges to $\phi$ in $L^{p}(\mathbf{R}), 1 \leq p \leq \infty$, and $l_{n} \rightarrow a /(\alpha-1)$, as $n \rightarrow \infty$, it follows that $\phi$ is supported in $[-a /(\alpha-1), a /(\alpha-1)]$.

\section{The Adjoint $W_{c K}^{*}$ And the Continuous Subdivision operator} by

$$
\left(W_{c}^{*} g\right)(x)=\int_{\mathbf{R}} \alpha c(\alpha y-x) g(y) d y, \quad g \in L^{q}(\mathbf{R}) .
$$

A straightforward calculation shows that

$$
\left(W_{c}^{*} g\right)(x)=\int_{-\frac{a}{\alpha}}^{\frac{a}{\alpha}} \alpha c(-\alpha y) g(x / \alpha-y) d y, \quad g \in L^{q}(\mathbf{R}) .
$$

Taking the Fourier transforms of the functions in (4.1) leads to

$$
\left(W_{c}^{*} g\right)^{\wedge}(u)=\alpha \hat{c}(-u) \hat{g}(\alpha u), \quad g \in L^{q}(\mathbf{R}) .
$$

A spectral radius formula for $r_{q}\left(W_{c}^{*}\right)$ is derived in [6].

Note that $L_{K}^{q}$ is not an invariant subspace of $W_{c}^{*}$ since the mapping $W_{c}^{*} f$ expands the support of $f$. In order to describe the adjoint of the restricted operator $W_{c, K}$, let

$$
\begin{aligned}
G(x) & :=\int_{-K}^{K} \alpha c(\alpha y-x) g(y) d y \\
& =\int_{\frac{x-a}{\alpha}}^{\frac{x+a}{\alpha}} \alpha c(\alpha y-x) g(y) d y, \quad x \in \mathbf{R} .
\end{aligned}
$$

The function $G$ is compactly supported with support in $[-a-\alpha K, a+\alpha K]$. Then

$$
\left(W_{c, K}^{*} g\right)(x)= \begin{cases}G(x), & x \in[-K, K], \\ 0, & \text { otherwise }\end{cases}
$$

In general, the simple relationship that exists between the Fourier transforms of $W_{c, K} f$ and $f$ is not available anymore for the conjugate operator $W_{c, K}^{*}$, but we do have

$$
\widehat{G}(u)=\alpha \hat{c}(-u) \hat{g}(\alpha u), \quad g \in L_{K}^{p} .
$$

The following results follow from Corollary 3.1 and the properties of adjoint operators.

Theorem 4.1. Suppose $c \in L^{1}(\mathbf{R})$ with $\operatorname{supp}(c) \subset[-a, a]$ and $\hat{c}(0)=1$. Then

$$
\sigma_{q}\left(W_{c, K}^{*}\right)=\left\{1 / \alpha^{k}: k=0,1, \ldots\right\} \cup\{0\} .
$$

The nonzero elements of the spectrum are simple eigenvalues of $W_{c, K}^{*}$. 
Note that the eigenfunctions $p_{n}$ of $W_{c, K}^{*}$ and the eigenfunctions $\phi^{(k)}$ of $W_{c, K}$ corresponding to the eigenvalues $1 / \alpha^{n}, n=0,1, \ldots$, form a biorthogonal pair of sequences. It is easy to see that $p_{0}(x)=1$. It was shown by Kabaya and Iri [8] that for the kernel

$$
c=\frac{1}{2(\alpha-1)} \chi_{[-\alpha+1, \alpha-1]},
$$

the eigenfunctions $p_{n}$ are polynomials. In general if $c \in L^{1}(\mathbf{R})$, they are not.

The adjoint operator $W_{c}^{*}$ is related to the continuous subdivision operator

$$
\left(S_{c} g\right)(x):=\int_{\mathbf{R}} \alpha c(x-\alpha y) g(y) d y, \quad g \in L^{q}(\mathbf{R}) .
$$

Clearly, $W_{\tilde{c}}^{*}=S_{c}$, where $\tilde{c}(x)=c(-x), x \in \mathbf{R}$. From an initial function $f_{0}$, the power iteration

$$
f_{n}:=S_{c}^{n} f_{0}, \quad n=0,1, \ldots,
$$

defines a continuous subdivision process (or integral subdivision process) studied in [4] and [3].

Let $D_{\alpha}: L^{q}(\mathbf{R}) \rightarrow L^{q}(\mathbf{R})$ be the dilation operator

$$
D_{\alpha} f(x):=\sqrt{\alpha} f(\alpha x)
$$

and define $\widetilde{W}_{\tilde{c}, K}^{*}: L_{K}^{q} \rightarrow L_{K}^{q}$ by

$$
\widetilde{W}_{\tilde{c}, K}^{*}:=D_{\alpha} W_{\tilde{c}, K}^{*} D_{\alpha} .
$$

Lemma 4.1. For any compactly supported $c \in L^{1}(\mathbf{R})$,

$$
\widetilde{W}_{\tilde{c}, K}^{*}=W_{c, K} .
$$

Proof. A straightforward substitution using (4.4), (4.5) and (4.9) gives

$$
\left(\widetilde{W}_{c, K}^{*}\right) g(x)=\int_{-K / \alpha}^{K / \alpha} \alpha^{2} c(\alpha y-\alpha x) g(\alpha y) d y, \quad g \in L_{K}^{q},
$$

for $x \in[-K, K]$. The relation (4.10) follows by a change of variable.

The following result on the convergence of the continuous subdivision process follows from Theorem 3.1.

Theorem 4.2. Suppose $\phi_{0}$ is a function in $L_{K}^{q}$ such that

$$
\hat{\phi}_{0}(0)=\cdots=\hat{\phi}_{0}^{(k-1)}(0)=0 \quad \text { and } \quad \hat{\phi}_{0}^{(k)}(0) \neq 0,
$$

for some nonnegative integer $k$. Then $\left(\alpha^{k} \widetilde{W}_{\tilde{c}, K}^{*}\right)^{n} \phi_{0}$ converges in the $L_{q}$-norm to an eigenfunction of $W_{c, K}$ corresponding to the eigenvalue $1 / \alpha^{k}$.

\section{REFERENCES}

1. C. D. Aliprantis and O. Burkinshaw, Principles of Real Analysis, Academic Press, San Diego, 1990. MR 91c:28002

2. C. K. Chui and X. Shi, Continuous two-scale equations and dyadic wavelets, Advances in Comp. Math. 2 (1994), 185-213. MR 95d:42038

3. W. Dahmen and C. A. Micchelli, Continuous refinement equations and subdivision, Advances in Comp. Math. 1 (1993), 1-37. MR 94h:41018

4. G. Derfel, N. Dyn, and D. Levin, Generalized functional equations and subdivision processes, J. Approx. Theory 80 (1995), 272-297. MR 95k:45003 
5. N. Dyn and A. Ron, Multiresolution analysis by infinitely differentiable compactly supported functions, Applied and Computational Harmonic Analysis 2 (1995), 15-20. MR 95k:42057

6. T. N. T. Goodman, C. A. Micchelli and J. D. Ward, Spectral radius formulas for the dilationconvolution integral operators, SEA Bull. Math. 19 (1995), 95-106. MR 96c:47042

7. K. Kabaya and M. Iri, Sum of uniformly distributed random variables and a family of nonanalytic $C^{\infty}$-functions, Japan J. Appl. Math. 4 (1987), 1-22. MR 89d:26023

8. K. Kabaya and M. Iri, On operators defining a family of nonanalytic $C^{\infty}$-functions, Japan J. Appl. Math. 5 (1988), 333-365.

9. V. A. Rvachev, Compactly supported solutions of functional-differential equations and their applications, Russian Mathematical Survey, 45:1 (1990), 87-120. MR 91j:34104

10. A. Sharma, Some simple properties of the up-function, Proc. Conf. at Aligarh (India) on Fourier Series, Approximation Theory and Applications (eds. Z. U. Ahmad, N. K. Govil, P. K. Jain), Wiley Eastern, New Delhi (to appear).

(R. Q. Jia and A. Sharma) Department of Mathematics, University of Alberta, Edmonton, Alberta, Canada T6G 2G1

E-mail address: jia@xihu.math.ualberta.ca

E-mail address: asharma@vega.math.ualberta.ca

(S. L. Lee) Department of Mathematics, National University of Singapore, 10 Kent Ridge Crescent, Singapore 0511

E-mail address: matleesl@haar.math.nus.sg 